\title{
Determination of heat exchange law using mean isotherm
}

\author{
Ilmars Iltins \\ Department of Engineering Mathematics \\ Riga Technical University \\ Riga, Latvia \\ iltins@inbox.lv
}

\author{
Marija Iltina \\ Department of Engineering Mathematics \\ Riga Technical University \\ Riga, Latvia \\ marijai@inbox.lv
}

\begin{abstract}
Determination of heat exchange between a solid and the environment is a significant inverse thermal physical problem. This heat exchange law irrespective of its nature can be defined easier if mean temperature of such solid is known. When mean temperature of the solid and speed of its change are known, it becomes possible to determine heat flow on the boundary of the solid. In its turn, when heat flow on the boundary, temperature on the boundary and ambient temperature are defined, heat exchange coefficient can be established. Therefore, the main attention is paid to a manner how mean temperature of a solid can be determined in the article. Examining inverse heat transfer problems, input data consist of measurements taken in temperature field as simple as possible mathematically in laboratory conditions. Hence, a symmetrical one-dimensional temperature field is discussed in the article.
\end{abstract}

Keywords-temperature field; inverse problem; series; heat conductivity; boundary conditions; isotherm.

\section{INTRODUCTION}

A symmetrical one-dimensional temperature field is described by the following equation

$$
\frac{\partial t}{\partial \tau}=a\left(\frac{\partial^{2} t}{\partial x^{2}}+\frac{k-1}{x} \frac{\partial t}{\partial x}\right)
$$

where $t$ is temperature, $\tau$ is time, $x$ is co-ordinate, $k=1$ is a plate, $k=2$ is a cylinder, $k=3$ is a sphere. If convective heat exchange occurs with the environment having time-dependent temperature $t_{e}(\tau)$ on the surface, then such boundary condition is valid

$$
\lambda \frac{\partial t(b, \tau)}{\partial \tau}=\alpha\left(t_{e}(\tau)-t(b, \tau)\right)
$$

where $\lambda$ is heat transfer coefficient, $\alpha$ is heat exchange coefficient, $b$ is a half of thickness in the case of a plate or radius in the case of a cylinder or a sphere. Initial conditions are the following

$$
t(x, 0)=t_{0} \text {. }
$$

Let us presume that initial temperature $t_{0}$ is constant. Discussing problem (1) - (3) is easier, by transferring to dimensionless values: $N=\frac{x}{b}$ is dimensionless co-ordinate, $N \in[-1 ; 1]$ if $k=1$ and $N \in[0 ; 1]$ if $k=2$ or $k=3, F=\frac{a \tau}{b^{2}}-$ is dimensionless time, $B=\frac{\alpha b}{\lambda}$ is dimensionless heat exchange coefficient, $T=\frac{t(x, \tau)-t_{0}}{t_{e}^{*}-t_{0}}$ is dimensionless temperature, $t_{e}^{*}=\max _{\tau \geq 0} t_{e}(\tau)$. Problem (1) - (3) in dimensionless form is written down as follows:

$$
\begin{aligned}
& \frac{\partial T}{\partial F}=\frac{\partial^{2} T}{\partial N^{2}}+\frac{k-1}{N} \frac{\partial T}{\partial N} \\
& \frac{\partial T(1, F)}{\partial N}=B\left(T_{e}(F)-T(1, F)\right) \\
& T(N, 0)=0 .
\end{aligned}
$$

Mean dimensionless temperature of a solid depends on dimensionless time $F$ and is calculated pursuant to formula [1]

$$
T_{v}(F)=k \int_{0}^{1} T(N, F) N^{k-1} d N
$$

If dependence of mean real temperature on time $t_{v}(\tau)$ is known, and it is always possible to calculate it, having established and temperature conductivity coefficient $a$, then heat flow on the boundary $x=b$ can be determined in accordance with formula [2]

$$
q(\tau)=c \rho \frac{b}{k} \frac{d t_{v}}{d \tau}
$$

where $c$ is heat capacity coefficient, and $\rho$ is density of material. As heat flow on the boundary $q(\tau)$ is the left side of boundary conditions (2), then after obtaining $q(\tau)$, ambient temperature $t_{e}(\tau)$, and temperature on the boundary $t(b, \tau)$, heat exchange coefficient $\alpha$ can be established from the boundary conditions (2). Here it should be noted that $t_{e}(\tau)$ 
can always be measured, $t(b, \tau)$ can be also measured in most cases. If $t(b, \tau)$ cannot be measured though, the [2] provides an approach how it can be calculated as per temperature measurements taken at an inner point $x_{1}<b$ of a solid.

\section{MEAN ISOTHERM}

In the [2] co-ordinate $N_{v}$ is called a mean isotherm at at which the following equality is true

$$
T\left(N_{v}, F\right)=T_{v}(F) \text {. }
$$

It follows from the last equation that $N_{v}$ depends on both dimensionless time $F, k$ and $B$. It is obvious that equation (9) has at least one root $N_{v} \in(0,1)$, because at least one root of equation

$$
\int_{0}^{1} N^{k-1} f(N) d N-f\left(N_{v}\right) \int_{0}^{1} N^{k-1} d N=0
$$

is located in the range $(0,1)$ if $f(N)$ is continuous. The [2] illustrates that if $T_{e}(F)$ tends to a constant quantity asymptotically when $F \rightarrow \infty$, solution $N_{v}$ of equation (9) does not depend practically on $F$ and $B$, and can be considered as a constant. In order to calculate this constant, solution of the problem (4) - (6) in the following form is used in the [2]

$$
T(N, F)=\sum_{n=0}^{\infty} T_{e}{ }^{(n)}(F) P_{n}(N, B, k),
$$

which is valid for sufficiently high $F$ values when influence of initial conditions can be not taken into account, $P_{n}(N, B, k)$, are co-ordinate functions mentioned in the [2]. Here we would like to note that the [2] is devoted to the process how a solution of heat transfer equation can be obtained in the form (11), by proving convergence rules of the series (11), and application of the series (11) in various inverse problems is discussed. For example, if $k=1$, [2] then

$$
\begin{gathered}
P_{0}(N, B, 1)=1 \\
P_{1}(N, B, 1)=\frac{N^{2}}{2}-\frac{1}{2} S_{1} \\
P_{2}(N, B, 1)=\frac{N^{4}}{24}-\frac{N^{2}}{4} S_{1}+\frac{1}{4}\left(S_{1}^{2}-\frac{1}{6} S_{2}\right) \\
P_{3}(N, B, 1)=\frac{N^{6}}{720}-\frac{N^{4}}{48} S_{1}+\frac{N^{2}}{8}\left(S_{1}^{2}-\frac{1}{6} S_{2}\right)- \\
-\frac{1}{8}\left(S_{1}^{3}-\frac{1}{3} S_{1} S_{2}+\frac{1}{90} S_{3}\right)
\end{gathered}
$$

where

$$
S_{m}=1+\frac{2 m}{B}
$$

Similar formulas [2] are valid if $k=2$ or $k=3$, which are not given here due to concision. Taking the first two addends from the series (11) and inserting them into the equation (9), we obtain that

$$
N_{v}=\sqrt{\frac{k}{k+2}}= \begin{cases}\frac{1}{\sqrt{3}}, & k=1 \\ \frac{1}{\sqrt{2}}, & k=2 . \\ \sqrt{\frac{3}{5}}, & k=3\end{cases}
$$

On the basis of properties of the series (11) provided in the [2], such substitution of the series by the first two addends is adequate only when $F$ value is high and $T_{e}(F)$ tends to a constant value asymptotically when $F \rightarrow \infty$. By taking three addends in the series (11), it is stated in the [2] that $N_{v}$ coordinate depends on $B$, but this dependence is weak, and namely, if $B$ changes from 0 to infinity, then $N_{v}$ changes from 0.577 to 0.559 if $k=1 ; N_{v}$ changes from 0.707 to 0.682 if $k=2$; and from 0.775 to 0.742 if $k=3$. Considering properties of the series (11) [2], as well as experience of practical calculations [3] and [4], the first three addends of the series (11) approximate precise solution of problem (4) - (6) for all $F$ values except $F$ values close to zero comparatively well. It means that the co-ordinate of the point where a temperature during entire heat transfer process is equal to mean temperature at a given moment of time is little dependent on type of boundary conditions and time as well. This suggests that if inserting thermocouple at a point with given co-ordinates, we will be able to read temperature with thermocouple not only at a particular point, but also mean temperature in a whole solid as these temperatures will approximately coincide. However, we cannot allege that the aforesaid presumption is correct as it is based on series approximation with the first three addends. Therefore, we specify the mathematical model in two ways. The first way: 5 addends are used in formula (11). The second way: formula (11) is not used and instead of that the problem (4)-(6) is solved numerically by means of software, by using a corresponding built-in function; then equation (9) is solved by using these data. Results thereof are discussed in the next paragraph.

\section{RESULTS}

If boundary conditions of the first type are set on the boundary

$$
T(1, F)=T_{e}(F)
$$

then solution of the problem (4), (13) can be put down as follows [2]

$$
T(N, F)=\sum_{n=0}^{\infty} T_{e}^{(n)}(F) P_{n}(N, k),
$$

but equation (9) can be re-written in the following way

$$
\sum_{n=0}^{\infty} T_{e}^{(n)}(F) P_{n}(N, k)=\sum_{n=0}^{\infty} T_{e}^{(n)} Q_{n}(k),
$$

where 


$$
Q_{n}(k)=k \int_{0}^{1} N^{k-1} P_{n}(N, k) d N .
$$

If $k=1$, then [2]

$$
\begin{gathered}
P_{0}(N, 1)=1 \\
P_{1}(N, 1)=\frac{N^{2}}{2}-\frac{1}{2} \\
P_{2}(N, 1)=\frac{N^{4}}{24}-\frac{N^{2}}{4}+\frac{5}{24} \\
P_{3}(N, 1)=\frac{N^{6}}{720}-\frac{N^{4}}{48}+\frac{5 N^{2}}{48}-\frac{61}{720} \\
P_{4}(N, 1)=\frac{N^{8}}{40320}-\frac{N^{6}}{1440}+\frac{5 N^{4}}{576}-\frac{61 N^{2}}{1440}+\frac{277}{8064}, \\
Q_{0}(1)=1, \quad Q_{1}(1)=-\frac{1}{3}, \quad Q_{2}(1)=\frac{2}{15,}, \\
Q_{3}(1)=-\frac{17}{315}, \quad Q_{4}(1)=\frac{62}{2835} .
\end{gathered}
$$

We have done many calculations at various boundary conditions, by using formula (15) with five addends for determination of $N_{v}$, as well as by using numerical solution of the problem (4), (13) at various $T_{e}(F)$. Table 1 summarizes some results if $k=1$.

Table 1

$N_{\mathrm{v}}$ minimum and maximum value if $F \in[100,1000]$

\begin{tabular}{|c|c|c|}
\hline Boundary conditions & {$\left[N_{v 1}, N_{v 2}\right]$ with } & {$\left[N_{v 1}, N_{v 2}\right]$} \\
$T_{e}(F)$ & formula $(15)$ & numerically \\
\hline $1-\cos (0.01 F)$ & {$[0.576914$,} & {$[0.576912$,} \\
& $0.57745]$ & $0.577462]$ \\
\hline $\sin (0.01 F)$ & {$[0.577251$,} & {$[0.577251$,} \\
& $0.577800]$ & $0.577768]$ \\
\hline $\sin (0.01 F)+\cos (0.01 F)$ & {$[0.576473$,} & {$[0.576475$,} \\
& $0.577651]$ & $0.578284]$ \\
\hline $1-\exp (-0.01 F)$ & 0.57734 & 0.577344 \\
\hline
\end{tabular}

It follows from the table that the point where a temperature during entire heat transfer process is equal to mean temperature at a given moment of time does not practically depend on time and boundary conditions and is approximately equal to 0.577 . Of course, all the above said cannot be considered as a proof to the fact that there is no boundary conditions were $N_{v}$ could differ from those illustrated here. In the case of any doubt, this calculation can be repeated for particular boundary conditions.

\section{CONCLUSIONS}

The calculations show that a point that does not practically depend on time in non-stationery symmetrical temperature field where temperature is equal to mean temperature inside whole solid at a particular moment of time exists. Temperature measurements at this point during non-stationery heat transfer process enable calculation of heat exchange coefficient in a simple way. Similarly, it follows from the calculations that a mathematical model of heat transfer process given in the [2] and not sufficiently studied in later papers, as well as undeservedly forgotten, is applicable in modelling of heat transfer process.

\section{REFERENCES}

[1] A. Likov, "Heat and mass transfer," Moscow: Energya, 1978. (in Russian)

[2] A. Temkin, "Inverse methods of thermal conductivity", Moscow: Energya, 1973. (in Russian)

[3] I. Iltins and M. Iltina, "One method of determination of thermal and physical characteristics of film-based materials," Scientific Proceedings of Riga Technical University, Computer Science, vol. 50, pp. 67-71, 2011.

[4] I. Iltins and M. Iltina, "Determination of heat physical characteristics by using series along boundary condition derivatives", WSEAS Press, Proceedings of the $2^{\text {nd }}$ International Conference on Mathematical Models for Engineering Science. Puerto De La Cruz, Tenerife, Spain, December 10-12, 2011.

\section{Creative Commons Attribution License 4.0 (Attribution 4.0 International, CC BY 4.0)}

This article is published under the terms of the Creative Commons Attribution License 4.0 https://creativecommons.org/licenses/by/4.0/deed.en US 